\title{
Prognostic Roles of Neutrophil-to-Lymphocyte Ratio and Stromal Tumor-Infiltrating Lymphocytes and Their Relationship in Locally Advanced Triple-Negative Breast Cancer Treated with Neoadjuvant Chemotherapy
}

\author{
Xue Dong ${ }^{a, b}$ Congfang Liu ${ }^{c}$ Jiaqi Yuan ${ }^{a, b}$ Shouman Wang ${ }^{a, b}$ \\ Nianhua Ding ${ }^{d}$ Yong Li $^{\mathrm{e}}$ Yuhui Wu ${ }^{\mathrm{a}, \mathrm{b}}$ Zhi Xiao ${ }^{\mathrm{a}, \mathrm{b}}$ \\ ${ }^{a}$ Department of Breast Surgery, Xiangya Hospital, Central South University, Changsha, China; \\ ${ }^{b}$ Clinical Research Center for Breast Cancer Control and Prevention in Human Province, Changsha, China; \\ ${ }^{\mathrm{C}}$ Health Management Center, Xiangya Hospital, Central South University, Changsha, China; ${ }^{\mathrm{d} D e p a r t m e n t}$ of Clinical \\ Laboratory, First Hospital of Changsha, Changsha, China; ${ }^{e}$ Department of Pathology, Xiangya Hospital, Central \\ South University, Changsha, China
}

\section{Keywords \\ Triple-negative breast cancer · Neoadjuvant chemotherapy · Neutrophil-to-lymphocyte ratio - Stromal tumor-infiltrating lymphocytes · Inflammatory marker · Prognosis}

\begin{abstract}
Introduction: The neutrophil-to-lymphocyte ratio (NLR) and stromal tumor-infiltrating lymphocytes (sTILs) are associated with immunogenicity and prognosis of patients with triple-negative breast cancer (TNBC). Objective: To investigated the prognostic roles of NLR and STILs and their relationship of TNBC patients treated with neoadjuvant chemotherapy (NAC). Methods: The clinical data of 170 patients with locally advanced TNBC who received NAC from January 2010 to December 2014 were collected. The difference among variables was calculated by $x^{2}$ test. The association between essential clinicopathological characteristics, pathological complete response ( $\mathrm{pCR}), \mathrm{NLR}$, and sTILs with disease-free survival (DFS) was analyzed. Kaplan-Meier and Cox analysis were performed to address the effects of clinical parameters on prognosis. Results: There was a trend that TNBC patients with lower baseline NLR (NLR1) or higher sTILs scoring would obtain a better $\mathrm{pCR}$ rate. NLR1 and sTILs were
\end{abstract}

not associated $(p>0.05)$. Patients with low NLR1 or high sTILs scoring had a significantly improved DFS compared to those with high NLR1 or low sTILs scoring ( $p=0.002$ and $p=0.001$, respectively). The increased lymphocyte count in peripheral blood after NAC was associated with the improved DFS outcome in both high and low NLR1 groups. Cox analysis revealed that NLR1 and STILs were independent prognostic predictors of DFS outcome $(p<0.001)$. Conclusion: Low NLR1 and high sTILs were associated with better DFS outcome in locally advanced TNBC patients treated with NAC. Further studies are needed to explore the connection between systemic and local inflammatory/immune markers.

(c) 2020 S. Karger AG, Basel

\section{Introduction}

Triple-negative breast cancer (TNBC) accounts for $10-15 \%$ of all breast cancers and possesses characteristics of aggressive invasiveness, easy recurrence and metastasis, and poor prognosis $[1,2]$. Due to the lack of expression of estrogen receptor (ER), progesterone receptor $(\mathrm{PR})$, and human epidermal growth factor receptor 2 (HER-2), chemotherapy is the standard-of-care treat- 
ment for TNBC. In addition, neoadjuvant chemotherapy (NAC) has recently been widely accepted for its helpfulness in terms of downsizing the tumor, evaluating the effectiveness of chemotherapy, and enhancing the rate of breast-conserving therapy [3]. TNBC patients who achieved pathological complete response (pCR) after NAC obtain much better survival benefit than those who had residual disease [4]. Recently, many studies have found that inflammation and immunity play a critical role in tumor initiation, malignant conversion, invasion, and metastasis [5]. Host anti-tumor immunity might offer a new strategy for curing TNBC which lacks treatment targets [6, 7]. The basic immune surveillance of the body, changes in systemic/local immune status, and the tumor microenvironment affect responses of therapy and even prognosis of breast cancer patients. Many studies explored the advantages of the neutrophil-to-lymphocyte ratio (NLR) and stromal tumor-infiltrating lymphocytes (sTILs) as predictive factors, and they have been reported to correlate with survival outcomes $[8,9]$, but only one study has investigated the relationship between NLR and TILs in locally advanced TNBC patients receiving NAC with a relatively small number of cases [10]. In this study, we retrospectively analyzed the NLR and sTILs as prognostic factors of 170 TNBC patients who were treated with NAC. The association between NLR and sTILs as inflammatory markers was also discussed to testify whether there was a consistency between systemic and local prognosis factors for TNBC patients.

\section{Method}

\section{Patients Background}

A total of 170 patients with invasive TNBC confirmed by pathological examination were successively collected. All patients had received NAC in the Breast Department, Xiangya Hospital, Central South University, Changsha, China, from January 2010 to December 2014. This study was approved by the Institutional Review Board of the Xiangya Hospital. Medical records were reviewed to collect and organize data on patients' age, sex, medical history, laboratory test, and pathologic results such as tumor size, lymph node status, hormone receptor status, and HER-2 status. All patients were treated with NAC of TEC regimens (docetaxel $75 \mathrm{mg} /$ $\mathrm{m}^{2} \mathrm{~d} 1$, epirubicin $75 \mathrm{mg} / \mathrm{m}^{2} \mathrm{~d} 1$, cyclophosphamide $500 \mathrm{mg} / \mathrm{m}^{2} \mathrm{~d} 1$, $1 / 21 d$ ), followed by modified radical mastectomy or breast-conserving surgery and axillary lymph node dissection, and then postsurgical radiotherapy. We excluded patients with inflammatory breast cancer, multiple tumors, acute and chronic injury, acute and chronic inflammation, hematological disorders, liver cirrhosis, and end-stage renal disease.

\section{Pathological Characteristics}

ER and PR were considered positive if there were at least $1 \%$ positive invasive tumor nuclei in the samples. HER-2 positive was defined as IHC staining of $3+$, IHC staining of $2+$, but FISH positive as HER-2/CEP17 ratio $>2.2$. TNBC patients were confirmed by pathology for negative ER, negative PR, and negative HER-2. pCR was defined as no evidence of residual invasive carcinoma in the breast, but residual ductal carcinoma in situ was included. Histopathologic evaluation of sTIL was performed on primary breast tumor tissues from a core needle biopsy, rather than intratumoral TILs, and was implemented according to the evaluation criteria recommended by the International TILs Working Group 2014 [11]. Each case was scored independently. Interpersonal discussions were allowed when there were differences in scoring. sTILs were evaluated within the borders of invasive tumors, and they were defined as area occupied by TILs over total stromal area. Those areas with TILs outside of the tumor border and around residual ductal carcinoma in situ and normal lobules were excluded, and areas with crush artifacts, necrosis, and regressive hyalinization were also excluded [12]. Evaluation of TILs in the stromal part of the tumor is demonstrated in Figure 1a. The results were scored as intervals: low sTILs scoring was defined as $0-10 \%$ (48 patients; Fig. 1b), middle sTILs scoring was defined as 11-20\% (48 patients; Fig. 1c), and high sTILs scoring was defined as $>20 \%$ (74 patients; Fig. 1c).

\section{Laboratory Data}

The NLR was defined as the absolute neutrophil count (N) divided by the absolute lymphocyte count $(\mathrm{L})$. The baseline NLR was defined as NLR1 which was calculated from the full blood count routinely performed within 1 week before the first cycle of chemotherapy. The median NLR1 value was 1.928, which was chosen to be the threshold. Patients were further divided into 2 groups, the low NLR1 group (NLR $\leq 1.928,84$ patients) and the high NLR1 group (NLR $>1.928,86$ patients). The second NLR was defined as NLR2 which was calculated from full blood count 1 month after radiotherapy. Change in lymphocyte count $(\Delta \mathrm{L})$ was defined as the absolute lymphocyte count (L1) within 1 week before the first cycle of chemotherapy minus the absolute lymphocyte count (L2) 1 month after radiotherapy. There were no signs of clinical infection, such as fever, on the day of blood sample collection.

\section{Statistical Analysis}

Pearson $\chi^{2}$ test was performed to compare categorical parameters of clinicopathological characteristics. For survival analyses, the endpoint was disease-free survival (DFS). DFS was defined as the length of time for TNBC patients to survive without any signs or symptoms of local relapse or metastasis after surgery. KaplanMeier curves and log-rank tests were performed to assess differences among groups. Multivariate Cox regression models were established to identify significant predictors of DFS. A $p<0.05$ was considered statistically significant. The results were reported including hazard ratios (HR) and 95\% CI. All analyses were carried out using SPSS version 17.0 for Windows (SPSS Inc., Chicago, IL, USA) as previously [13].

\section{Results}

\section{Clinicopathological Characteristics of 170 Patients with TNBC}

The clinical and pathological data subset used for this analysis was described according to different NLR1 or sTILs groups. There were 84 patients in the low NLR1 group and 86 patients in the high NLR1 group. The number of patients in the low, middle, and high sTILs groups were 48,48 , and 74 , respectively. The median follow-up was 34 months (range, 6-74 months). Detailed distributions of patients with different age, tumor size, lymph 
a

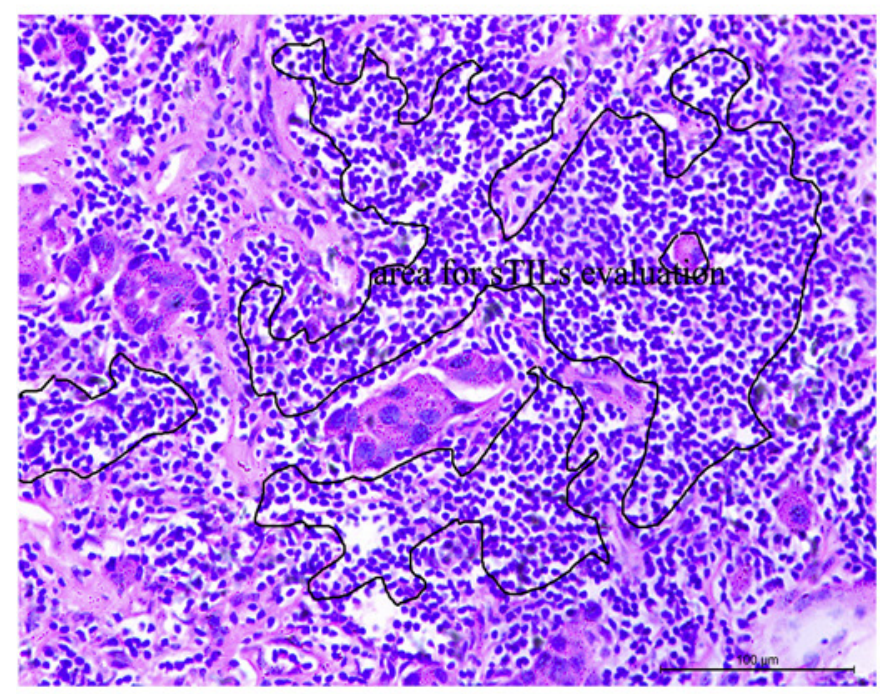

c

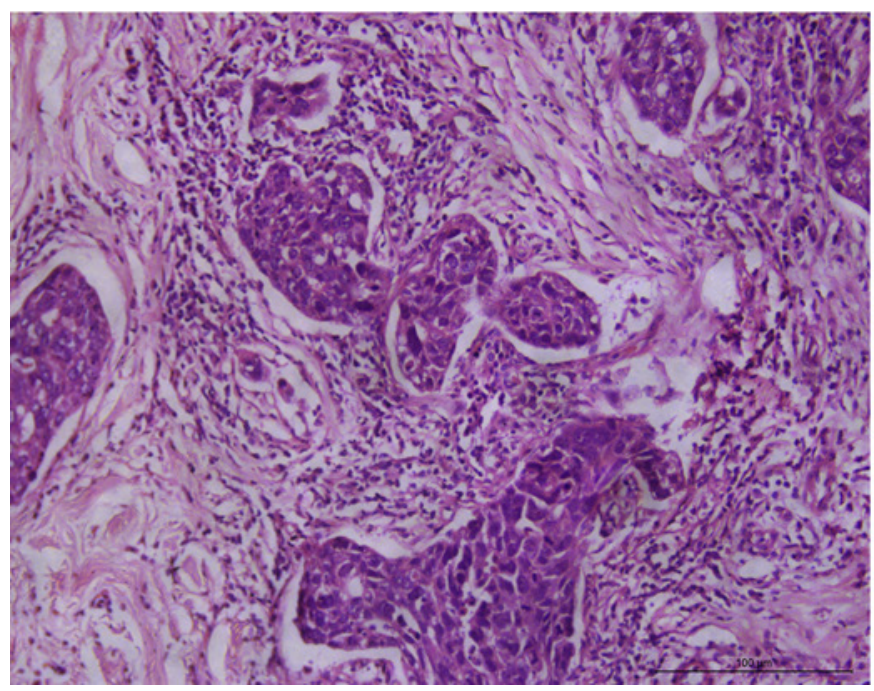

b

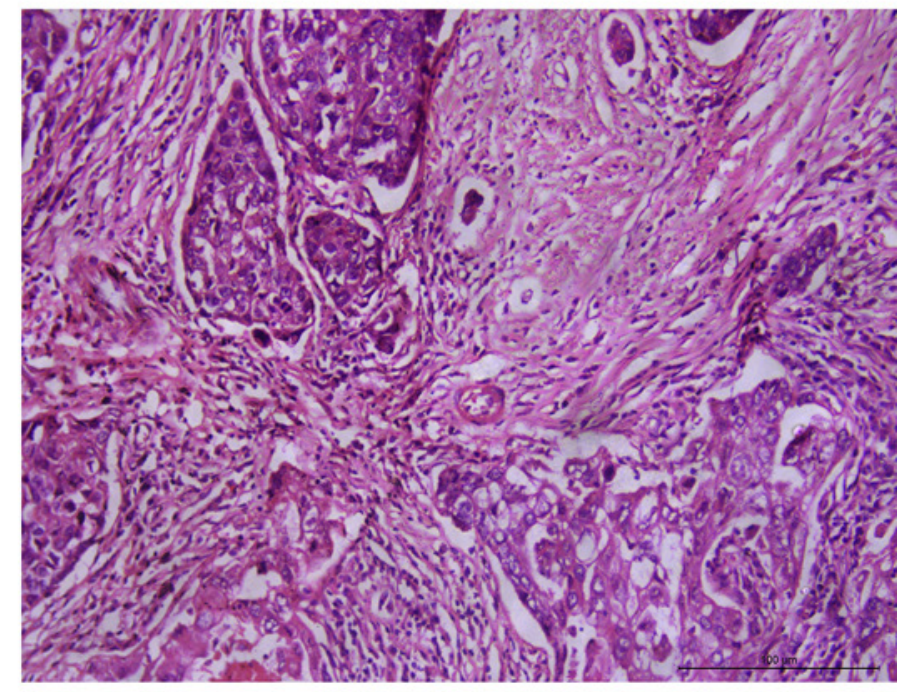

d

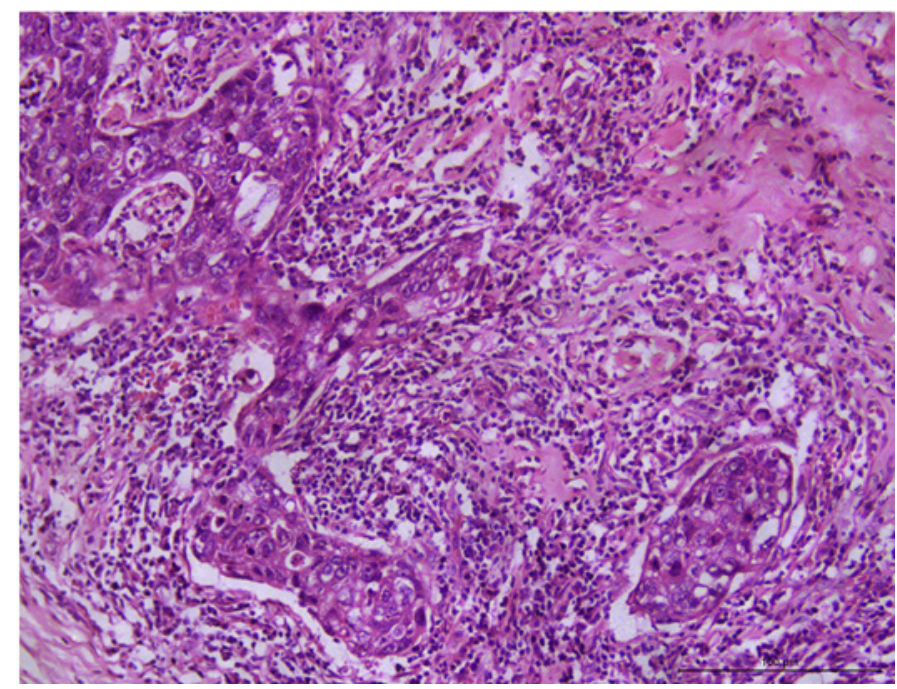

Fig. 1. Evaluation of sTILs in TNBC. a Assessment of sTILs. sTILs were reported for the stromal compartment ( = \% sTILs). b Low sTILs scoring represented $0-10 \%$ of sTILs. c Middle sTILs scoring represented 11-20\% of sTILs. d High sTILs scoring represented $>20 \%$ of sTILs.

node status, and histological grade were summarized in Table 1. There was no significant difference in age, tumor size, lymph node status, and histological grade between low and high NLR1 groups, or among different sTILs groups $(p>0.05)$.

\section{The Response to NAC}

The primary tumors shrunk more obviously if TNBC patients had a lower NLR1 value. However, there was no significant difference in the pCR rate between low NLR1 group and high NLR1 group, but the pCR rate in low NLR1 group was higher than that in high NLR1 group (33.3 vs. $23.3 \%, p>0.05$; Table 2). There was no significant correlation of the pCR rate after NAC among different sTILs groups, but with the sTILs score rising up, the pCR rate increased gradually ( 20.8 vs. $25.0 \%$ vs. $35.1 \%, p>0.05$ ).

\section{The Relationship between NLR1 and sTILs}

The blood NLR1 and tumor sTILs were chosen to represent systemic and local inflammatory status, and the relationship between NLR1 and sTILs was analyzed at the time of diagnosis of invasive disease. However, there was no significant correlation between NLR1 and sTILs ( $p>$ 0.05; Table 3).

\section{Survival Analysis}

Patients with high NLR1 showed a significantly lower 5-year DFS rate than patients with low NLR1 (69.8 vs. 
Table 1. Comparison of clinicopathological characteristics of TNBC patients in different NLR1 and sTILs groups

\begin{tabular}{|c|c|c|c|c|c|c|c|}
\hline Characteristics & $\begin{array}{l}\text { Low NLR1 } \\
\text { patients }\end{array}$ & $\begin{array}{l}\text { High NLR1 } \\
\text { patients }\end{array}$ & $p$ value & $\begin{array}{l}\text { Low sTILs } \\
\text { patients }\end{array}$ & $\begin{array}{l}\text { Middle sTILs } \\
\text { patients }\end{array}$ & $\begin{array}{l}\text { High sTILs } \\
\text { patients }\end{array}$ & $p$ value \\
\hline \multicolumn{8}{|l|}{ Age, years, $n(\%)$} \\
\hline$\leq 40$ & $16(53.3)$ & $14(46.7)$ & \multirow[t]{2}{*}{0.636} & $6(20.0)$ & $8(26.7)$ & $16(53.3)$ & \multirow[t]{2}{*}{0.425} \\
\hline$>40$ & $68(48.6)$ & $72(51.4)$ & & $42(30.0)$ & $40(28.6)$ & $58(41.4)$ & \\
\hline \multicolumn{8}{|l|}{$\mathrm{T}, \mathrm{cm}, n(\%)$} \\
\hline$\leq 5$ & $52(47.3)$ & $58(52.7)$ & \multirow[t]{2}{*}{0.450} & $26(23.6)$ & $30(27.3)$ & $54(49.1)$ & \multirow[t]{2}{*}{0.098} \\
\hline$>5$ & $32(53.3)$ & $28(46.7)$ & & $22(36.7)$ & $18(30.0)$ & $20(33.3)$ & \\
\hline \multicolumn{8}{|l|}{$\mathrm{LN}, n(\%)$} \\
\hline $\mathrm{pN} 1-2$ & $68(50.0)$ & $68(50.0)$ & \multirow[t]{2}{*}{0.759} & $42(30.9)$ & $34(25.0)$ & $60(44.1)$ & \multirow[t]{2}{*}{0.119} \\
\hline $\mathrm{pN} 3$ & $16(47.1)$ & 18 (52.9) & & $6(17.6)$ & $14(41.2)$ & $14(41.2)$ & \\
\hline \multicolumn{8}{|l|}{ Grade, $n(\%)$} \\
\hline II & $22(45.8)$ & $26(54.2)$ & \multirow[t]{2}{*}{0.558} & $14(29.2)$ & $8(16.7)$ & $26(54.2)$ & \multirow[t]{2}{*}{0.085} \\
\hline III & $62(50.8)$ & $60(49.2)$ & & $34(27.9)$ & $40(32.8)$ & $48(39.3)$ & \\
\hline Total & 84 & 86 & & 48 & 48 & 74 & \\
\hline
\end{tabular}

NLR1 and sTILs were not significantly associated with clinical pathological factors.

Table 2. The correlation of pCR with NLR1 and sTILs after NAC

\begin{tabular}{|c|c|c|c|}
\hline & Non-pCR patients & pCR patients & $p$ value \\
\hline \multicolumn{4}{|c|}{ NLR1, $n(\%)$} \\
\hline Low & $56(66.7)$ & $28(33.3)$ & 0.144 \\
\hline High & $66(76.7)$ & $20(23.3)$ & \\
\hline \multicolumn{4}{|l|}{ sTILs, $n(\%)$} \\
\hline Low & $38(79.2)$ & $10(20.8)$ & 0.194 \\
\hline Middle & $36(75.0)$ & $12(25.0)$ & \\
\hline High & $48(64.9)$ & $26(35.1)$ & \\
\hline Total & 122 & 48 & \\
\hline
\end{tabular}
NAC.

NLR1 or sTILs were not significantly associated with pCR after

$90.5 \%, p=0.002$; Fig. 2a). Nevertheless, patients with different NLR2 values which were obtained 1 month after radiotherapy showed no significant difference in DFS (data not shown). The effect of changes in lymphocyte count on DFS was analyzed. In the high NLR1 group, patients with an increase of lymphocyte count $(\Delta \mathrm{L}=\mathrm{L} 1-\mathrm{L} 2$ $\leq 0)$ showed significantly higher DFS than patients with a decrease in lymphocyte count $(\Delta \mathrm{L}=\mathrm{L} 1-\mathrm{L} 2>0 ; p=0.031$; Fig. 2b). In the low NLR1 group, there was the same trend as that in the high NLR1 group. However, there was no significant difference between them as only 2 patients had an increase in lymphocyte count (Fig. 2c). Patients in the high sTILs group showed a significantly higher 5-year DFS rate than patients in the low sTILs group (91.9 vs. $22.8 \%, p=0.001$; Fig. $2 \mathrm{~d}$ ).

Multivariate Cox analysis showed that lymph node metastases $(\geq 10)$, higher histological grade, non-pCR,
Table 3. The correlation of NLR1 in whole blood with sTILs in local tumor microenvironment

\begin{tabular}{|c|c|c|c|}
\hline & $\begin{array}{l}\text { Low NLR1 } \\
\text { patients }\end{array}$ & $\begin{array}{l}\text { High NLR1 } \\
\text { patients }\end{array}$ & $p$ value \\
\hline \multicolumn{4}{|l|}{ sTILs, $n(\%)$} \\
\hline Low & $22(45.8)$ & $26(54.2)$ & \\
\hline Middle & $26(54.2)$ & $22(45.8)$ & 0.706 \\
\hline High & $36(48.6)$ & $38(51.4)$ & \\
\hline Total & 84 & 86 & \\
\hline
\end{tabular}

The lack of significant correlation among baseline NLR and sTILs.

higher NLR1, and lower sTILs were independent prognostic indicators of poor prognosis in patients with TNBC $(p<0.05)$. Primary tumor size was not an independent prognostic factor for TNBC patients undergoing NAC $(p=0.273$; Table 4$)$.

\section{Discussion}

In this study, we analyzed the effect of some conventional prognostic factors, such as tumor size, nodal involvement, tumor grade, and $\mathrm{pCR}$, and 2 inflammatory predictors, such as NLR and sTILs, on the DFS among patients with locally advanced TNBC who underwent NAC. Besides the normal predictors of nodal involvement, tumor grade and pCR, the NLR1 and sTILs are associated with worse prognosis in TNBC patients. 


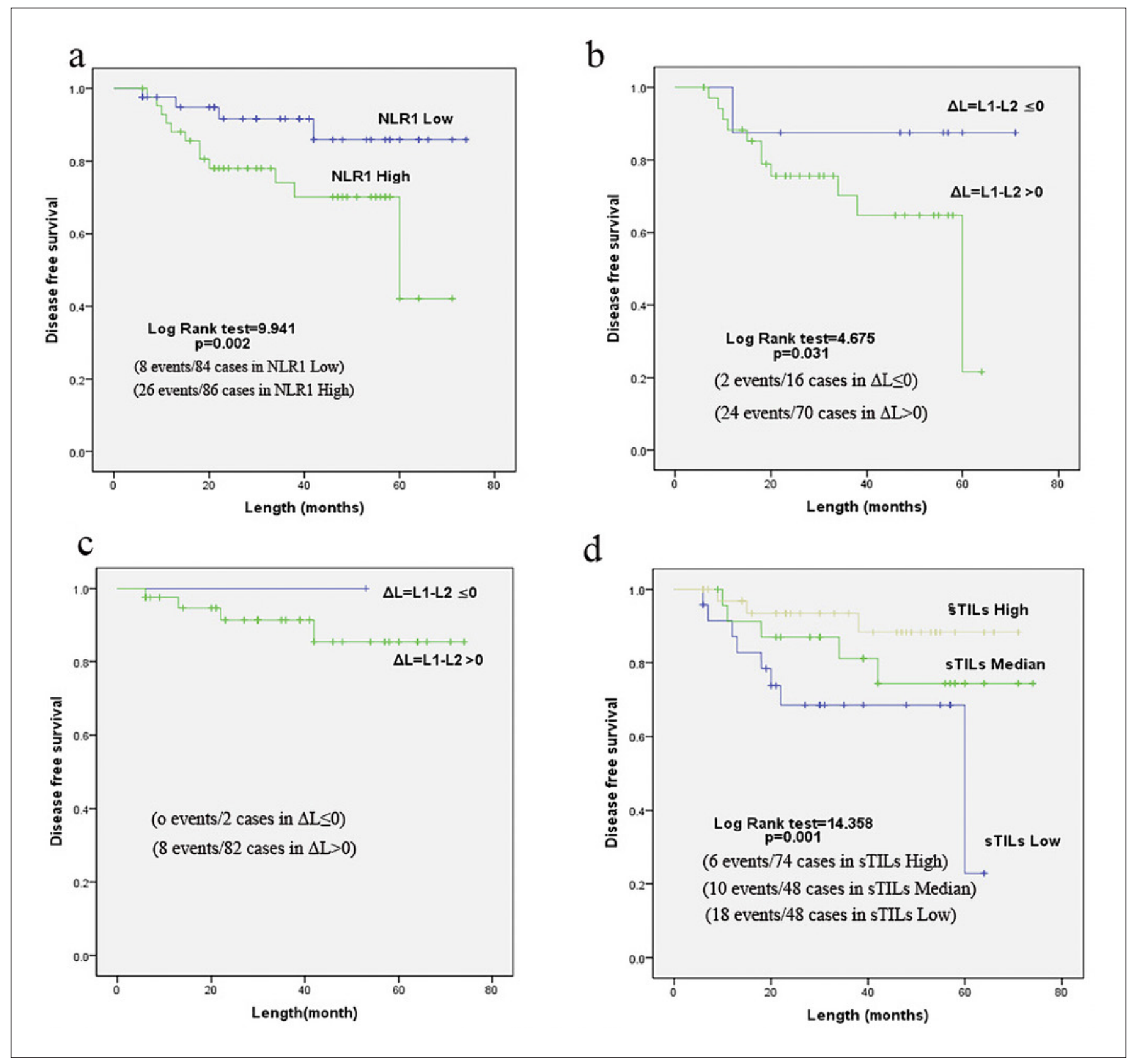

Fig. 2. Kaplan-Meier curves for DFS outcomes based on NLR1, and the changes of lymphocyte count and sTILs. Patients in the low NLR1 group (a) or in the high sTILs group (d) had an improved 5-year DFS ( $p=0.002$ and $p=0.001$, respectively). The increase in lymphocyte count was correlated with a better DFS outcome in the low NLR1 group ( $\mathbf{b} ; p=0.031)$, and seemed to link to a better DFS outcome in the high NLR1 group (c).

NLR is a routinely available marker of the systemic inflammatory response. The presence of higher NLR in the blood has been identified as a poor prognostic factor in various cancers, such as gastrointestinal cancer, non-small cell lung cancer, hepatocellular carcinoma, and breast cancer [14-16]. Due to the lack of treatment targets and poor prognosis, TNBC raises more attention of immune evaluation and therapies. We investigated the association between NAC response and NLR1 of TNBC patients based on our hospital database. Our data suggested that the tu- mor in patients with low NLR1 shrunk more obviously than those with high NLR1, and this was in line with some previous studies $[17,18]$. While there was a trend that the pCR rate in the low NLR1 group was higher than that in the high NLR1 group (33.3 vs. $23.3 \%$ ), there was no significant difference in the pCR rate between them. This was, at least partly, consistent with data from other studies that showed a significantly higher $\mathrm{pCR}$ rate in low NLR groups than in high NLR groups of TNBC patients $[17,18]$. For the survival analysis, our results were in line with the com- 
Table 4. Multivariate Cox analysis of the influence of clinicopathological factors on DFS

\begin{tabular}{|c|c|c|c|}
\hline \multirow[t]{2}{*}{ Parameters } & \multicolumn{3}{|l|}{ DFS } \\
\hline & HR & $95 \%$ CI & p value \\
\hline \multicolumn{4}{|c|}{ Tumor size, $\mathrm{cm}$} \\
\hline$\leq 5$ & 1 & & \\
\hline$>5$ & 1.541 & $0.711-3.337$ & 0.273 \\
\hline \multicolumn{4}{|l|}{ Lymph node } \\
\hline $\mathrm{pN} 1-2$ & 1 & & \\
\hline $\mathrm{pN} 3$ & 3.899 & $1.421-10.693$ & 0.008 \\
\hline \multicolumn{4}{|l|}{ Grade } \\
\hline II & 1 & & \\
\hline III & 6.509 & $2.048-20.681$ & 0.001 \\
\hline \multicolumn{4}{|l|}{$\mathrm{pCR}$} \\
\hline Yes & 1 & & \\
\hline No & 6.648 & $2.027-21.808$ & 0.002 \\
\hline \multicolumn{4}{|l|}{ NLR1 } \\
\hline Low & 1 & & \\
\hline High & 6.910 & $2.712-17.609$ & $<0.001$ \\
\hline \multicolumn{4}{|l|}{ sTILs } \\
\hline High & 1 & & \\
\hline Middle & 1.923 & $0.673-5.494$ & 0.222 \\
\hline Low & 7.501 & $2.565-21.936$ & $<0.001$ \\
\hline
\end{tabular}

Multivariate analysis of correlation among clinicopathological factors and DFS. HR, hazard ratio; CI, confidence interval.

mon viewpoint that low NLR is associated with better DFS among TNBC patients $[16,19]$. Although the threshold value of NLR was different among various studies, generally, low NLR was associated with better tumor response to chemotherapy and better survival among TNBC patients $[16,19]$. We also investigated the effect of changes of inflammatory markers on survival outcomes. Data from our results showed that it was not the changes of NLR but the lymphocyte count that affected the DFS outcomes, whether in the high NLR1 group or the low NLR1 group. This was not consistent with the only result - at least to our knowledge - of a retrospective study with 50 TNBC patients which showed that modest change of NLR after NAC $(-30 \%<$ NLR change $<100 \%)$ was associated with prolonged DFS [10]. Our study and the study mentioned above both had retrospectively collected locally advanced TNBC patients. However, these 2 studies did differ in the time point to collect the blood for laboratory analysis as well as in the chemotherapy regimens the patients received.

Previous literatures showed that high sTILs are associated with favorable survival in breast cancer, especially in TNBC, and this again was confirmed by our results $[8,9]$. Some limitations in evaluation of sTILs existed in this study. First, threshold values of 10 and $20 \%$ were used to divide patients with different sTILs into 3 groups (low, middle, and high). However, the working group's consensus was that sTILs might provide more biological rel- evant information when scored as a continuous variable [11]. Second, available evidence of sTILs evaluation after NAC in residual disease, especially in case of pCR, is not inadequate to date [12]. If the changes of sTILs after NAC could be effectively evaluated, they might provide more information concerning survival outcomes.

The mechanisms of anti-tumor immunity are not yet well understood. Neutrophils are recognized as not only pro-tumor effectors which might promote tumor cell proliferation, produce proangiogenic factors, and enhance tumor cell invasiveness, but also inhibitors of activity of T cells and natural killer cells [20-22]. Lymphocytes are important factors of immune surveillance and anti-tumor response $[23,24]$. The effects of circulating lymphocytes or localized lymphocytes in the microenvironment of the tumor on prognosis of TNBC have been emphasized in many studies, and its prospective mechanisms might be involved in immunosuppression of Foxp3+ regulatory $\mathrm{T}$ cells and the presence of a high number of CD8+ T cells and cytotoxic cells $[8,16,25,26]$. But the relationship between circulating and localized lymphocytes has not been fully studied.

In the situation of bacterial infection or sepsis, systemic inflammatory status and response commonly correlated with the severity of local infection [27]. We supposed that systemic inflammatory status might be associated with local inflammatory response to tumor. To verify this hypothesis, we chose blood NLR and tumor sTILs to represent systemic and local inflammatory status, and analyzed the relationship between NLR and sTILs at the time of diagnosis of invasive disease. However, there was no significant association between NLR and sTILs found in our study. Another study concerning systemic and local inflammatory changes during NAC had analyzed the relationship between changes in serum NLR with tumor TIL changes. There were trends for negative correlation between changes in TILs and serum NLR during NAC, but also without statistical significance [10]. Based on the data from our studies, it is difficult to verify that systemic inflammatory status could reflect the local inflammatory response to invasive tumor. Systemic or local response to invasive TNBC may be more similar to chronic inflammatory diseases, in which it is difficult to detect any changes of inflammatory cell count in blood. This might be explained by immune tolerance which was manipulated by invasive tumors to avoid elimination by the host immune system [28, 29]. Some cytokines, transcription factors, and cell surface receptors should be investigated to interpret the association between systemic and local inflammation as well.

In conclusion, this study showed that low NLR1 is associated with better chemotherapy response and better DFS outcome in locally advanced TNBC patients receiving NAC. Higher sTILs in the primary tumor was associated with better DFS. NLR, sTILs, nodal involvement, tumor grade, and pCR were independently associated with 
DFS. No significant correlation was found between the systemic inflammatory marker of NLR and the local inflammatory marker of sTILs. Further studies are needed for the exploration of connection between systemic and local inflammatory/immune markers.

\section{Statement of Ethics}

All procedures involving human participants, which were performed in this study, were in accordance with the ethical standards of the institutional and/or national research committee and with the 1964 Helsinki Declaration and its later amendments or comparable ethical standards. This article does not contain any studies on animals performed by any of the authors. Informed consent was obtained from all individual participants included in the study.

\section{Conflict of Interest Statement}

The authors have no conflicts of interest to disclose.

\section{Funding Sources}

Dr. Yuhui Wu is funded by Natural Science Foundation of Hunan Province (S2019JJKWLH0198).

\section{Author Contributions}

X.D. collected the data and wrote the manuscript. C.L. collected the data. J.Y. collected the survival data. S.W. and N.D. did the analysis of data. Y.L. analyzed and scored the sTILs. Y.W. designed the study. Z.X. designed the study, scored the sTILs, and reviewed the manuscript.

\section{References}

1 Bray F, Ferlay J, Soerjomataram I, Siegel RL, Torre LA, Jemal A. Global cancer statistics 2018: GLOBOCAN estimates of incidence and mortality worldwide for 36 cancers in 185 countries. CA Cancer J Clin. 2018 Nov;68(6):394-424.

2 Liao L, Song M, Li X, Tang L, Zhang T, Zhang L, et al. E3 Ubiquitin Ligase UBR5 Drives the Growth and Metastasis of Triple-Negative Breast Cancer. Cancer Res. 2017 Apr;77(8): 2090-101.

3 Chaudhary LN, Wilkinson KH, Kong A. Triple-Negative Breast Cancer: Who Should Receive Neoadjuvant Chemotherapy? Surg Oncol Clin N Am. 2018 Jan;27(1):141-53.

4 Liedtke C, Mazouni C, Hess KR, André F, Tordai A, Mejia JA, et al. Response to neoadjuvant therapy and long-term survival in patients with triple-negative breast cancer. J Clin Oncol. 2008 Mar;26(8):1275-81.

5 Grivennikov SI, Greten FR, Karin M. Immunity, inflammation, and cancer. Cell. 2010 Mar;140(6):883-99.

6 Spitzer MH, Carmi Y, Reticker-Flynn NE, Kwek SS, Madhireddy D, Martins MM, et al. Systemic Immunity Is Required for Effective Cancer Immunotherapy. Cell. 2017 Jan 26; 168(3):487-502.e15

7 Jia H, Truica CI, Wang B, Wang Y, Ren X, Harvey HA, et al. Immunotherapy for triplenegative breast cancer: Existing challenges and exciting prospects. Drug Resist Updat. 2017 May;32:1-15.

8 Denkert C, von Minckwitz G, Darb-Esfahani S, Lederer B, Heppner BI, Weber KE, et al. Tumour-infiltrating lymphocytes and prognosis in different subtypes of breast cancer: a pooled analysis of 3771 patients treated with neoadjuvant therapy. Lancet Oncol. 2018 Jan; 19(1):40-50.

9 Pistelli M, De Lisa M, Ballatore Z, Caramanti M, Pagliacci A, Battelli N, et al. Pre-treatment neutrophil to lymphocyte ratio may be a useful tool in predicting survival in early triple negative breast cancer patients. BMC Cancer. 2015 Mar; 15(1):195.

10 Lee J, Kim DM, Lee A. Prognostic Role and Clinical Association of Tumor-Infiltrating Lymphocyte, Programmed Death Ligand-1 Ex- pression with Neutrophil-Lymphocyte Ratio in Locally Advanced Triple-Negative Breast Cancer. Cancer Res Treat. 2019 Apr;51(2):649-63.

11 Salgado R, Denkert C, Demaria S, Sirtaine N, Klauschen F, Pruneri G, et al. The evaluation of tumor-infiltrating lymphocytes (TILs) in breast cancer: recommendations by an International TILs Working Group 2014. Ann Oncol. 2015 Feb;26(2):259-71.

12 Dieci MV, Radosevic-Robin N, Fineberg S, van den Eynden, Ternes N, Penault-Llorca F, et al. Update on tumor-infiltrating lymohocytes (TILs) in breast cancer, including recommendations to assess TILs in residual disease after neoadjuvant therapy and in carcinama in situ: A report of the International Immuno-Oncology Biomaker Working Group on Breast Cancer. Semin Cancer Biol. 2018 Oct;52(Pt 2):16-25.

13 Ding N, Huang T, Yuan J, Mao J, Duan Y, Liao $\mathrm{W}$, et al. Yes-associated protein expression in paired primary and local recurrent breast cancer and its clinical significance. Curr Probl Cancer. 2019 Oct;43(5):429-37.

14 Guthrie GJ, Charles KA, Roxburgh CS, Horgan PG, McMillan DC, Clarke SJ. The systemic inflammation-based neutrophil-lymphocyte ratio: experience in patients with cancer. Crit Rev Oncol Hematol. 2013 Oct;88(1): 218-30.

15 Shimada H, Takiguchi N, Kainuma O, Soda H, Ikeda A, Cho A, et al. High preoperative neutrophil-lymphocyte ratio predicts poor survival in patients with gastric cancer. Gastric Cancer. 2010 Aug;13(3):170-6.

16 Ethier JL, Desautels D, Templeton A, Shah PS, Amir E. Prognostic role of neutrophil-tolymphocyte ratio in breast cancer: a systematic review and meta-analysis. Breast Cancer Res. 2017 Jan;19(1):2.

17 Asano Y, Kashiwagi S, Onoda N, Noda S, Kawajiri H, Takashima $\mathrm{T}$, et al. Predictive Value of Neutrophil/Lymphocyte Ratio for Efficacy of Preoperative Chemotherapy in Triple-Negative Breast Cancer. Ann Surg Oncol. 2016 Apr;23(4):1104-10.

18 Chae S, Kang KM, Kim HJ, Kang E, Park SY, Kim JH, et al. Neutrophil-lymphocyte ratio predicts response to chemotherapy in triplenegative breast cancer. Curr Oncol. 2018 Apr; 25(2):e113-9.

19 Wei B, Yao M, Xing C, Wang W, Yao J, Hong $\mathrm{Y}$, et al. The neutrophil lymphocyte ratio is associated with breast cancer prognosis: an updated systematic review and meta-analysis. OncoTargets Ther. 2016 Sep;9:5567-75.

20 Coussens LM, Werb Z. Inflammation and cancer. Nature. 2020 Dec;420(6917):860-7.

21 DeNardo DG, Coussens LM. Inflammation and breast cancer. Balancing immune response: crosstalk between adaptive and innate immune cells during breast cancer progression. Breast Cancer Res. 2007;9(4):212.

22 El-Hag A, Clark RA. Immunosuppression by activated human neutrophils. Dependence on the myeloperoxidase system. J Immunol. 1987 Oct;139(7):2460-13.

23 Shankaran V, Ikeda H, Bruce AT, White JM, Swanson PE, Old LJ, Schreiber RD. IFNgamma and Iymphocytes prevent primary tumour development and shape tumour immunogenicity. Nature. 2001 Apr;410(6832):1107-11.

24 Savas P, Salgado R, Denkert C, Sotiriou C, Darcy PK, Smyth MJ, et al. Clinical relevance of host immunity in breast cancer: from TILs to the clinic. Nat Rev Clin Oncol. 2016 Apr; 13(4):228-41.

25 Mahmoud SM, Paish EC, Powe DG, Macmillan RD, Grainge MJ, Lee AH, et al. Tumorinfiltrating CD8+ lymphocytes predict clinical outcome in breast cancer. J Clin Oncol. 2011 May;29(15):1949-55.

26 Mahmoud SM, Paish EC, Powe DG, Macmillan RD, Lee AH, Ellis IO, et al. An evaluation of the clinical significance of FOXP3+ infiltrating cells in human breast cancer. Breast Cancer Res Treat. 2011 May;127(1):99-108.

27 Meisner M. Update on procalcitonin measurements. Ann Lab Med. 2014 Jul;34(4): 263-73.

28 Lu L, Barbi J, Pan F. The regulation of immune tolerance by FOXP3. Nat Rev Immunol. 2017 Nov;17(11):703-17.

29 Joyce JA, Fearon DT. T cell exclusion, immune privilege, and the tumor microenvironment. Science. 2015 Apr;348(6230):74-80. 